\section{UNA PUBLICACIÓN COMPROMETIDA CON LA FORMACIÓN DEL PROFESORADO DE ENSEÑANZA SECUNDARIA: LA SEGUNDA ENSEÑANZA}

\author{
Miryam Carreño \\ Teresa Rabazas \\ Universidad Complutense de Madrid
}

\section{A FACULTY TRAINING COMMITTED PUBLICATION: LA SEGUNDA ENSEÑANZA [THE SECONDARY EDUCATION]}

\begin{abstract}
In this article, is analized the issue of high school teachers pedagogical training. For this aim, we have used as main source the review "La Segunda Enseñanza" published between the years 1922 and 1928 en el Instituto San Isidro de Madrid (Spain). The study shows how some state high school teachers concerned about improving their pedagogical knowledge.
\end{abstract}

KEY WORDS: Review La Segunda Enseñanza; Instituto San Isidro; José Rogerio Sánchez; pedagogical innovation.

\section{EVOLUCIÓN Y PROPÓSITO DE ESTA PUBLICACIÓN}

La Segunda Enseñanza comenzó a editarse en 1922 en el instituto San Isidro de Madrid. Se trata de la primera publicación dirigida al profesorado de la enseñanza secundaria en España. En el año en que se inicia su edición, su director es el profesor José Rogerio Sánchez, catedrático del citado instituto, quien estará al frente de la revista hasta 1927. El consejo de redacción está formado, en este momento, por los catedráticos de instituto Miguel Adellac, Miguel Aguayo, Francisco Barnés, José Estalella, Vicente García de Diego, Manuel Manzanares, Manuel de Sandoval y Luis Ollés'.

En el año 1923 se suspendió su publicación ${ }^{2}$ que se reanudó en 1924, con el nombre de Revista de Segunda Enseñanza, que se mantendrá hasta 1928, año en que es sustituida por El Instituto, de periodicidad trimestral. En la presentación del primer número de esta nueva versión de la revista, se perfilan con bastante precisión las dificultades que tuvieron que afrontar quienes se habian
RESUMEN: En este artículo se analiza el contenido de la revista $L a$ Segunda Enseñanza, publicada entre los años 1922 y 1928 en el instituto San Isidro de Madrid, dirigida especialmente a los profesores de la entonces denominada segunda enseñanza. Se han estudiado, en primer lugar, las ideas contenidas en sus páginas, acerca de la necesidad de la formación pedagógica de los profesores. También se ha indagado acerca del contenido de sus artículos en lo referente a orientaciones didácticas para los profesores de este nivel.

PALABRAS CLAVE: Revista La Segunda Enseñanza; Instituto San Isidro; José Rogerio Sánchez; innovaciones pedagógicas.

empeñado en sostener este medio de comunicación. Alli se explica la desaparición de la revista que le precedió -Revista de Segunda Enseñanza- que, según se dice, a pesar del "aprecio que le tenía el profesorado le faltó el apoyo económico". Como "consecuencia inevitable de esa falta de colaboración por parte de gran número de nuestros compañeros" se produjo "la desaparición lamentable de esta Revista" (El Instituto, 1928, n. 1 1, 1). Su corta vida se debería, pues, al escaso respaldo institucional $y, \sin d u d a$, al poco interés que su contenido despertó entre la mayor parte de los docentes de este nivel ${ }^{3}$.

Estas son las circunstancias en que nace El Instituto que pretende "recoger la antorcha, aún encendida, que la mano de mi digno compañero -se refiere a Rogerio Sánchez- no podía ya, bien a pesar suyo, sostener" (Ibidem). Así fue como el profesorado del instituto San Isidro, dirigido en ese momento por el profesor don Miguel Aguayo, acordó de manera unánime continuar la publicación de la revista con el nuevo título antes aludido. Piensan que de esta forma se proporcionará a "nuestros compañeros de toda España el 
medio de comunicación espiritual que ha estado a punto de faltarles..." (Ibidem).

Este entusiasmo inicial decae pronto. El retraso en la publicación de los números del tercer y cuarto trimestre es significativo en este sentido. Una breve presentación en la página inicial de estos números, expone las razones por las cuales se ha decidido poner fin a la edición de la revista. "Una excelente intención y un móvil sentimental hizo aparecer el primer número de esta publicación" pero "fue escaso el número de los colaboradores y, por tanto, nuestro esfuerzo excede a nuestras posibilidades" (Ibidem, 129). Otras razones vinculadas a la situación vivida entonces por la segunda enseñanza, son esgrimidas por la redacción para justificar el cierre: "La vida de los Institutos españoles de hoy -dicen- reducidas a tres años de escolaridad ${ }^{4}[. .$.$] , hace absolutamente ineficaz toda labor$ de orientación espiritual colectiva [...]. EL INSTITUTo en vista de todo esto y de algo más que sería inútil apuntar, da por terminada su misión; se despide de sus colaboradores y lectores y reconoce paladinamente la esterilidad de sus afanes" (Ibidem, 130).

Termina así la vida de la revista que analizamos, la cual transcurre entre 1922 y 1928 con la discontinuidad que supone la ausencia de la publicación en el año 1923.

El propósito que guía esta publicación se va manifestando en los números iniciales del año 1922. En el primer número se alude a la conveniencia de que España tenga una publicación dedicada a la segunda enseñanza con la cual "pueda ponerse a tono con las numerosas y excelentes que en Francia, en Bélgica, en Italia, en Alemania, en Estados Unidos, etc., etc., sostienen los profesores de la enseñanza media" (La Segunda Enseñanza, 1922, n. 1, 3). La comparación con otros países europeos es bastante frecuente en sus páginas lo que nos permite apreciar la existencia de, al menos, una cierta comunicación entre los profesores españoles y sus colegas europeos.

En el $n .{ }^{\circ} 2$ se vuelve sobre la finalidad perseguida y se aclara que "esta revista será en todo momento un eco de las aspiraciones de la Asociación de Catedráticos de Instituto pero, por hoy, no es órgano de la Asociación ni aspira a recibir de ella como entidad ningún auxilio". Se trata, por lo tanto, de "una iniciativa puramente particular, así si ella fracasare, nada que a la colectividad importe como obra suya habrá fracasado"5. En el n. 5 del año 1922, se vuelve a hablar de la finalidad que se persigue en un breve artículo firmado por Rogerio Sánchez:

La Segunda Enseñanza ha nacido, vive y vivirá, Dios mediante, con absoluta independencia de criterio en todas aquellas cuestiones que "están entregadas a las disputas de los hombres". Aún en ellas todos sus colaboradores tendrán, a buen seguro, la discreción y prudencia que las relaciones sociales imponen y los respetos que son debidos a la opinión ajena. Cuestiones discutibles son todas las que a la enseñanza nacional, oficial o privada corresponden, y ni suspicacias ni insinuaciones tendrán fuerza para desviarla del fin propuesto. Es él, y creíamos que no había necesidad de repetirlo, formar un estado de opinión en pro de la renovación de los estudios del bachillerato, de su organización y disciplina, en lo cual, no solamente puede estar interesado el profesor oficial, sino todo ciudadano español capaz de discernimiento en estas cuestiones. Por eso nuestras páginas están a disposición de cuantos poniéndose por encima de intereses materiales, si ellos no están perfectamente justificados, quieran intervenir en nuestra obra (La Segunda Enseñanza, 1922, n. ${ }^{\circ}$, 242-243).

Si tenemos en cuenta que el momento de su aparición es una época de reformas y que los debates con este motivo son frecuentes y acalorados, se la presenta, también, como "obra de paz y de concordia, que no podrá desarrollarse sino en el ambiente propicio para que los nobles ideales se desenvuelvan". Por eso mismo "no viene con posiciones tomadas, ni ha de vivir en torre inexpugnable: todos los criterios con alteza de mira sustentados, por radicales que sean, aquí tendrán acogida". Por otra parte, "ha de mirar y examinar, como objeto inmediato, cuanto a los estudios del bachillerato se refiere: las múltiples cuestiones que al profesorado de este grado de enseñanza importan; la evolución de nuestros planes; métodos y procedimientos docentes; todo, en fin, lo que a la mayor eficacia de la cultura general interesa" (La Segunda Enseñanza, 1922, n. 1, 1-2).

El carácter abierto y la búsqueda de diálogo que estos profesores pretenden darle a su trabajo se explicita con claridad en el primer número en cuyo artículo inicial, titulado "Significación de esta revista", admiten la estrecha relación de este nivel con la enseñanza primaria que es "su base sustancial y por eso desea un cambio de opiniones, siempre que sea posible, con el magisterio español" (Ibidem, 1). 


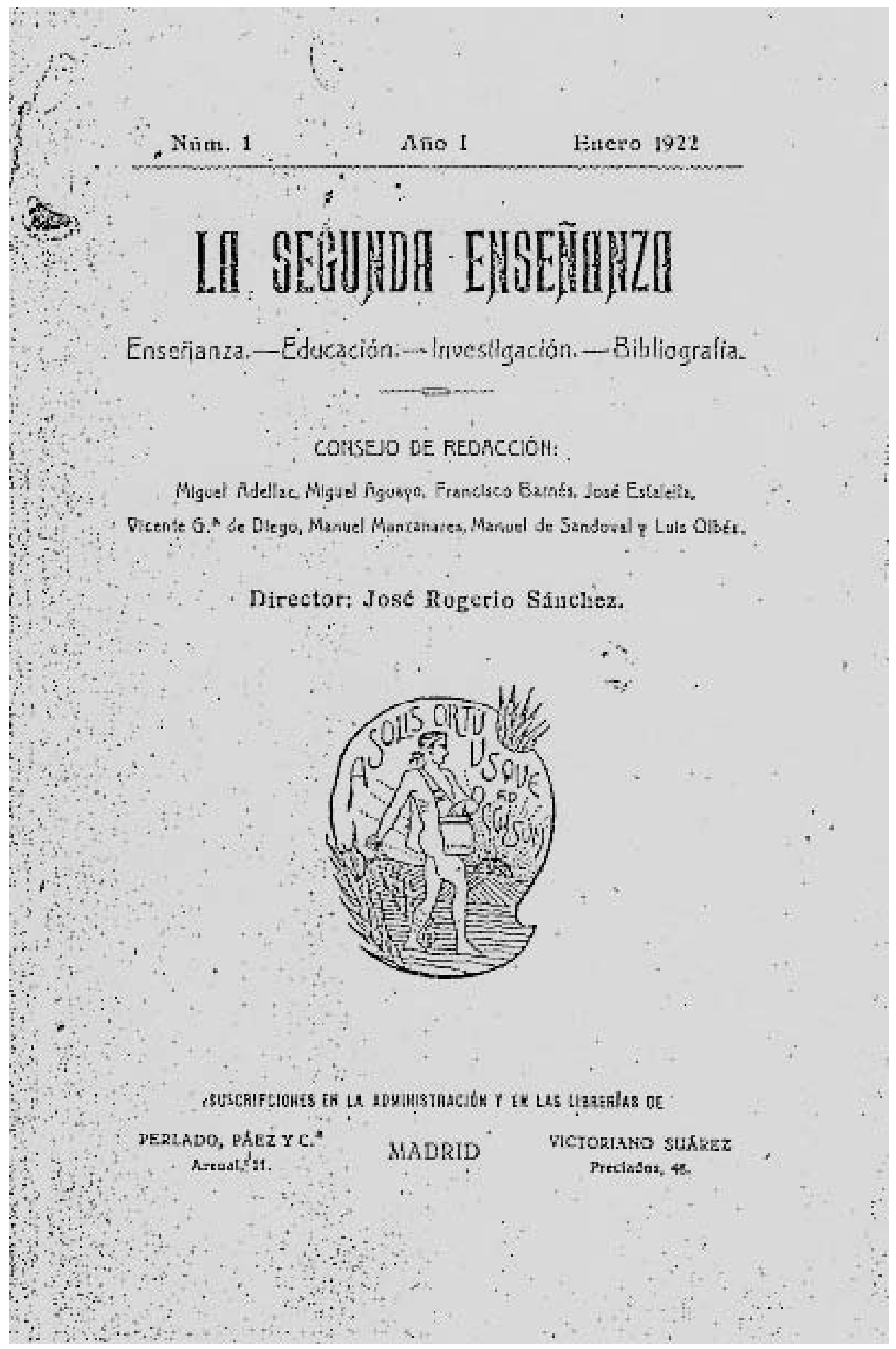


Es claro, pues, el propósito, que guía esta publicación: servir de canal de comunicación y formación pedagógica del profesorado público, con la intención de plantear sus pensamientos, estrategias docentes y sugerencias en un ámbito de consideración y respeto hacia las opiniones de este colectivo.

Nos parece interesante hacer mención al elogio que mereció tanto esta obra como su director en el Congreso Internacional de Luxemburgo. Uno de los congresistas saludó la aparición de La Segunda Enseñanza diciendo: "Los números publicados por la interesante revista han sido leídos con gran placer; los artículos son variados y muy interesantes [...], la revista reúne una materia abundante y preciosa y puede asegurarse, que hoy, es la mejor que los estudios secundarios tienen en el mundo" (La Segunda Enseñanza, 1922, n. $7,370-371$ ).

\section{EL CONTEXTO}

\section{La política educativa}

Parece oportuno realizar algunas consideraciones sobre las circunstancias en que surge La Segunda Enseñanza. Es época de inestabilidad política caracterizada por lo que algunos historiadores denominan "conservadurismo tradicional". Como señala Puelles Benitez: "Desde 1902 a 1923 se suceden atropelladamente 39 presidentes de Gobierno y 53 ministros de Instrucción Pública" (Puelles Benitez, 1999, 223).

En este marco político tornadizo y variable, la política educativa no puede ser sino un reflejo de aquél. Las reformas emprendidas por los gobiernos -siempre reformas desde arriba- tenían una vida muy corta que terminaba, casi sin excepciones, en fracaso. En el año en que surge la revista aquí analizada está vigente la reforma de Romanones (RD de 17 de agosto de 1901), cuyo propósito fundamental se orientaba a corregir el pésimo estado de la enseñanza oficial $^{6}$. En particular, en lo que respecta al nivel de la segunda enseñanza, Romanones se lamenta de la existencia de unos institutos despoblados y desnudos de ciencia que compiten con unos colegios religiosos repletos cuyas aulas daban amparo al clericalismo, por otra parte, vehículo del odio liberal.
El citado RD de 1901 organiza la segunda enseñanza transformando los centros estatales de bachillerato en Institutos Generales y Técnicos. El art. 1 del citado RD establece que los institutos de segunda enseñanza darán los siguientes estudios: estudios generales de bachillerato, estudios elementales y superiores de magisterio, estudios elementales de agricultura, industria, comercio, bellas artes y enseñanzas nocturnas para obreros. Los de bachillerato se impartirian en seis cursos. Señala el profesor Puelles que esta reforma estaba orientada por la "idea de hacer de los institutos un semillero de cultura humanística y científica, un haz complejo de centros clásicos y modernos" $(1999,214)$. Como expresó el mismo Romanones, intentaba "cambiar la orientación de la segunda enseñanza disponiendo que los estudios técnicos se desenvolvieran al compás de los clásicos y que sirvieran de preparación para las carreras industriales las enseñanzas dadas en los institutos". (Cit. Puelles, 1999, 214). Fue un plan original pero duramente atacado por la derecha católica; tampoco tuvo la aceptación de la izquierda. Para Romanones, sin embargo, el fracaso se debió a la oposición de los profesores: "La medida no arraigó, pues el profesorado oficial resistió a aquel injerto, fue lástima pues la idea bien planteada, podría tener eficacia" (Ibidem, 215).

Otro aspecto a tener en cuenta en la educación de este período, es que está enmarcada en la tensión constante entre conservadores y liberales. Los enfrentamientos más importantes se refieren a la enseñanza de la religión, la libertad de enseñanza, las titulaciones del profesorado. A pesar de ello hay aspectos en que las ideas liberales se mantienen o ganan terreno; por ejemplo, la idea de Estado-docente no se discute en lo que respecta a la educación primaria, cuya obligatoriedad ejercida y controlada por el Estado es plenamente aceptada; tampoco es motivo de excesivo cuestionamiento el monopolio de éste sobre la Universidad. Sin embargo, en la enseñanza secundaria el intervencionismo del Estado retrocede. Confirman lo dicho determinadas normativas, como el RD de 3 de febrero de 1910 que limita la intervención del Estado en los establecimientos privados concediéndole competencias sólo para inspeccionar la higiene de los locales y para impedir todo aquello considerado contrario a la moral. En el mismo sentido, puede mencionarse, entre otras medidas, la RO de 15 de octubre de 1914 por la que no se exigirá título a los profesores de determinadas órdenes religiosas. Se perdió así una de las reivindicaciones más queridas de los 
liberales: la exigencia de titulación para ejercer la docencia en la segunda enseñanza.

La reforma de Eduardo Callejo de 25 de agosto de 1926, se lleva a cabo dentro del período de existencia de la revista que analizamos. Por entonces se denominaba, como ya dijimos, Revista de Segunda Enseñanza. En el número 25 se reproducen los Reales Decretos del mes de agosto referidos a la reforma de la estructura y del plan de estudios de este nivel de enseñanza y el referido a los libros de texto. Consideran necesario realizar esta publicación porque la revista debe ser: "Un archivo de información para futuras épocas" (Revista de Segunda Enseñanza, 1926, n. $25,305-311)$.

En lo que respecta al nivel que estudiamos, el denominado Plan Callejo estableció una división del bachillerato en dos ciclos: el bachillerato elemental de tres años -novedad en Europa-, un año común a las dos secciones de ciencias y letras y dos de bachillerato universitario, es decir, una extensión de seis años, igual que en los planes anteriores, pero inferior a los que dedicaban a este nivel otras naciones europeas (Díaz de la Guardia, 1988, 376-416).

Otros aspectos a destacar de esta reforma son: la obligatoriedad de la religión, el uso exclusivo de los manuales declarados de texto, la prohibición de la enseñanza en lengua distinta al castellano, la exigencia de vigilar que no se impartan doctrinas opuestas a la unidad de la patria y ofensivas a la religión, entre otras.

Además del contexto normativo propio de la segunda enseñanza, tiene interés recordar los intentos de reforma de la universidad que se produjeron en el período estudiado, ya que también de ellos se hace eco la revista analizada. Durante el ministerio de César Silió se promulgó el RD de 21 de mayo de 1919 por el que se establecía la primera fase de la autonomía universitaria que sería suspendida poco después, en 1922, como consecuencia de las resistencias que el decreto generó; la derogación, sin embargo, tuvo un carácter totalmente legal pues esta norma contradecía preceptos de leyes aún vigentes, como la Ley de Instrucción Pública de 1857.

Durante la dictadura de Primo de Rivera (1923-1929) se inicia otra reforma universitaria cuyo marco legal es el Decreto-Ley de 19 de marzo de 1928. Esta reforma desencadenó la protesta de estudiantes y profesores como consecuencia de que el artículo 53 de la mencionada norma, sobre relación entre enseñanza oficial y privada "inspirado por el obispo Leopoldo Eijo Garay y el Director de enseñanza superior, Gonzalo Oliveros, equiparaba el colegio de Jesuitas de Deusto y el de Agustinos de El Escorial a las universidades del Estado en la expedición de títulos académicos" (Tuñón de Lara, 1981, 202). Ante la oleada de protestas que no cesó a pesar de las medidas de fuerza del gobierno, éste se vio obligado a derogar dicho artículo en 1929.

\section{El entorno pedagógico}

En lo que respecta al ámbito estrictamente pedagógico, La Segunda Enseñanza puede contextualizarse dentro del ambiente de preocupación y exigencia de renovación pedagógica que ciertos grupos de intelectuales mantuvieron desde las últimas décadas del siglo XIX. Sin duda, como se ha señalado repetidamente, esta preocupación reformista en el ámbito educativo tiene su más claro antecedente en el pensamiento de Francisco Giner de los Ríos y en su realización más significada, la Institución Libre de Enseñanza (ILE), de presencia e influencia indudables en el período de vida de la revista que aquí se analiza. Los profesores, agrupados en torno a esta institución, fueron construyendo un discurso sobre la segunda enseñanza en el que la formación pedagógica del profesorado secundario fue un tema destacado. Los textos, cuyo contenido alude a la necesidad de reforma de este nivel, fueron escritos en los últimos años del siglo XIX y primeros del XX?.

En el Congreso Pedagógico de 1892 se presentó a debate la cuestión de la segunda enseñanza, sin duda por influencia de los institucionistas, que ocupó la sección segunda del Congreso. El séptimo tema de discusión de dicha sección, hace referencia a la formación y selección del profesorado de este nivel. En la sesión del dia 15 de octubre de 1892 se aprobó por unanimidad, a modo de recomendación, una proposición que expresaba la conveniencia de "crear una Escuela especial pedagógica donde los Profesores, después de terminar la carrera, aprendiesen el difícil arte de enseñar" (Actas Congreso, 1892, 50).

Un hecho de gran significación en este sentido fue la creación del Instituto-Escuela, una iniciativa de renovación 
y modernización de la enseñanza que situó a España al mismo nivel que el de otros paises europeos y que marcó un hito en la historia de las innovaciones educativas españolas. Esta institución, creada en 1918 durante el ministerio de Santiago Alba, tuvo entre sus finalidades la formación del futuro personal docente de la segunda enseñanza. Este ensayo pedagógico intentó abordar "los problemas centrales y más delicados de la segunda enseñanza; entre ellos, la formación del futuro personal docente secundario" (Díaz de la Guardia, 1988, 313-339), que formaba parte importante del ideario institucionista. Giner de los Ríos había afirmado que "la formación de un maestro, de cualquier orden y grado que sea, no se alcanza con una enseñanza pura y exclusivamente teórica, ni con sólo los principios generales de aquella ciencia sino que ha de ser a la vez, como la de todo arte, doctrinal y práctica"8. Por otra parte, como señala el profesor A. Viñao: "El fracaso de las reformas de 1868 y 1873 y, en general, la experiencia vivida en el sexenio democrático habían convencido a los institucionistas de la inutilidad de emprender cualquier reforma sin contar con profesores adecuadamente formados e imbuidos del espíritu e ideas de la misma" (Viñao Frago, 2000, 77).

Como puede apreciarse por lo dicho antes, la aspiración de una formación pedagógica para el profesorado de la segunda enseñanza tiene ya una larga tradición cuando comienza a publicarse la revista que analizamos, en el año 1922. Sin embargo, la tónica dominante durante el primer tercio del siglo XX es la reticencia hacia este tipo de formación. En opinión de la mayoría del profesorado secundario, lo importante era el conocimiento de la materia a enseñar no los aspectos metodológicos.

El alejamiento de los profesores de secundaria de toda preocupación pedagógica confiere singularidad a la revista que estudiamos, especie de punta de lanza en este tema en lo que respecta al profesorado de los institutos públicos. En referencia a esta cuestión, es muy significativo el comentario que se hace en el Boletín Escolar y que la revista recoge en un apartado titulado "Lo que piensan de nosotros". Alli se expresa el asombro que causa una publicación de carácter pedagógico en el ámbito de los catedráticos de instituto:

El hecho de publicar una revista pedagógica los catedráticos de Instituto es muy significativo, porque se creía que en la enseñanza secundaria no era necesaria la Pedagogía, sólo indispensable a los maestros, espíritus menos formados, y que a los catedráticos de la segunda enseñanza les bastaba con su saber o cultura.

Vemos con gusto que va modificando algo este parecer. Indudablemente, tarde o temprano, las barreras que separan los tres grados de la enseñanza oficial caerán y muchos asuntos, que interesan por igual, a todo el profesorado serán estudiados y resueltos en común por maestros y catedráticos (La Segunda Enseñanza, 1922, n. 2 , 100).

\section{IDEAS En La SEgunda ENSEÑANZA SOBRE LA NECESIDAd DE LA FORMACIÓN PEDAGÓGICA DEL PROFESORADO SECUNDARIO}

A pesar de ese clima de indiferencia al que se aludía antes, a veces animadversión, Rogerio Sánchez y el grupo de profesores que forman el equipo de redacción de La Segunda Enseñanza emprenden su propia campaña a favor de la formación del profesorado secundario con la mirada puesta en sus compañeros de la enseñanza pública. La revista se transforma entonces en el vehículo transmisor de sus ideas y de las del pequeño grupo de profesores que están de acuerdo a este respecto. En sus páginas aparecen varios artículos argumentando sobre la necesidad de la formación pedagógica; puede comprobarse esta tendencia en repetidas ocasiones durante el período de su existencia.

Así, en el primer número, encontramos un artículo firmado por el ministro de Instrucción Pública y Bellas Artes, César Silió, titulado "Pedagogía nacional". En este texto el autor pone en evidencia la necesidad de una reforma educativa. Se refiere al "atraso" de España que, para este ministro, "tiene una sola raíz: nuestra deficiente y desmedrada educación [...] y una sola terapéutica: la implantación de una pedagogía nacional [que] bien dirigida y persistentemente continuada, obraría el milagro de renovar nuestra alma" (Silió, 1922, n. 1, 4).

Un artículo como el citado antes, que inicia el discurrir de La Segunda Enseñanza, no deja lugar a dudas sobre el interés del grupo de profesores que sostenía esta publicación con respecto a la necesidad de la formación pedagógica. 
Muy significativo para el tema que venimos analizando resulta un artículo de Miguel Adellac, catedrático del instituto Cardenal Cisneros, también publicado en la revista. Para este profesor, el docente de secundaria es "escultor de almas" y es, por eso, la docencia, oficio delicado que necesita de tres condiciones principales: "Cultura general, preparación profesional y amor al oficio".

Quiero hablar en estas líneas de la segunda condición, toda vez que suele ser entre nosotros la que menos atenciones merece. La experiencia revela, además, que tienen todos o casi todos nuestros colegas conocimientos más que sobrados para encargarse de enseñar una o varias materias de las que constituyen el plan de la segunda enseñanza en España.

La dificultad radica en el cómo y el cuánto de tal enseñanza; es decir en el procedimiento y en la extensión. Para algunos la Pedagogía es cosa baladi o invención de pedantes. Quien así discurre cree sin duda que se nace maestro y no se considera este noble oficio nuestro como todos los demás, necesitados de aprendizaje (Adellac, 1922, n. ${ }^{0}$ 1, 5).

Es, pues, para este profesor, el conocimiento pedagógico el que nos permitiría plantearnos algunas cuestiones básicas sobre el cómo y el cuánto, es decir, enseñar para que se nos entienda y saber poner el límite a lo que enseñamos.

Para Adellac este proceso formador debiera llevarse dentro de los institutos. Realiza la propuesta de un proyecto que es: "Perfectamente gacetable si se realiza de la siguiente forma":

Supongamos que tres, cuatro Institutos, recibieran ayudantes-alumnos, Doctores o Licenciados en Ciencias y Letras, pensionados por el Ministerio y mediante oposición o propuesta de las Universidades de su procedencia. Algo análogo a los becarios de la Escuela Superior del Magisterio durante su periodo de estudio en ella.

Estos ayudantes-alumnos asistirian al Instituto durante el número de cursos que se determinase (dos o tres), donde cada semana el catedrático numerario explicaría una lección-modelo, y cada ayudante otra lección que prepararia con el tiempo necesario, verificando su práctica en la clase oficial, ante el director del establecimiento, el catedrático y los demás ayudantes-alumnos.

Concluida la lección, vendria la crítica. Primero la de sus compañeros; después la del catedrático; resumiría el di- rector, o en su defecto el catedrático más antiguo de la Sección.

¿Es que no hay en España Institutos capaces de realizar esta función preparatoria de candidatos a su Profesorado? (lbidem, 7-8).

Esta propuesta dirigida a los ayudantes -a quienes considera la verdadera cantera del futuro profesorado de institutos-, constituye, sin lugar a dudas, un modelo para dicha formación en el que la práctica tiene un papel central. Demuestra el interés de algunos docentes de la enseñanza pública por llenar el vacío que la falta de los conocimientos pedagógicos dejaba en la carrera docente. La pregunta con que finaliza la cita anterior deja claro, además, la firme creencia en la posibilidad de que esta tarea formativa se podía llevar a cabo en el ámbito de la educación pública.

Especial importancia tiene, para el análisis aquí realizado, un artículo que comienza a publicarse en el n. 4 de la revista con el nombre de "Sentido pedagógico del bachillerato"; se publica en dos entregas y su autoría corresponde a Rogerio Sánchez. Alli, el autor comienza recordando que las Facultades de Filosofía y Letras fueron creadas en España para que constituyesen "la más alta expresión de lo que pudiéramos Ilamar liberalidad científica; su fin ideal debía ser el cultivo de la Ciencia por la Ciencia misma". Sin embargo, la realidad se fue construyendo de manera muy distinta y las "disciplinas liberales se convirtieron en carreras profesionales [...]. En resumen: la Ciencia se convirtió en profesión" (Sánchez, 1922, n. ${ }^{\circ} 4$, 226). Por eso se comprende fácilmente que "lo que diriamos los medios y recursos pedagógicos no habian de ser, ni tenían por qué, objeto de propósito encajado entre las disciplinas de aquellas dos facultades universitarias" (Ibidem). Pero "de lo soñado a lo real -señala- hubo gran diferencia", de manera que, en resumen, la Ciencia se convirtió en profesión. Sin embargo, éste no es el problema, afirma, siempre y cuando cada científico realice la adaptación necesaria para la profesión elegida. $Y$ en esta necesaria adaptación:

Sólo una excepción absurda tiene esto que podríamos llamar necesidad universal: cuando el licenciado o doctor en ciencias o letras no pudo vivir en el campo de la investigación o de la especulación científica, o pudiendo no quiso, o queriendo no tenía aptitud para ello, asióse, o naturalmente derivó hacia una profesión, y la más socorrida fue la de maestro, es decir, se convirtió en un profesional.

ARBOR Vol. 187749 mayo-junio [2011] 485-500 ISSN: 0210-1963 
Parecía oportuno que al decidirse por este derrotero, la sociedad, que le había de confiar pocos o muchos discípulos, especialmente en edad de formación mental, hubiera exigido a este nuevo profesional garantía de aptitud, de técnica, de manipulación...

Pues nada de eso. Para la única profesión en la cual no se exige -ni aún hoy- en España, curso de práctica es para ejercer de catedrático (Ibidem, 227).

Esta falta de formación, para el autor, impide una relación adecuada entre profesor y alumno. Para restablecerla "es menester que el profesor sepa lo que es un niño en ese difícil tránsito en que va a aparecer un joven". Es muy claro en su apoyo a los conocimientos pedagógicos por parte del profesor de la segunda enseñanza cuando afirma que si éste "no ama su objeto directo del trabajo, que no es precisamente la ciencia, sino el discípulo, debe abandonar su profesión, dedicarse a la alta investigación, al comercio, a la política, al tresillo, a cualquier cosa que no sea la enseñanza en este grado delicadísimo que llamamos la Segunda Enseñanza" (Sánchez, 1922, n. ${ }^{\circ}$ 6, 335). De esta manera, reitera la necesidad de adaptación por parte del profesor para adquirir una cultura pedagógica que "hoy por hoy, es lo que no se hace en nuestra patria" (Ibidem, 337).

Para este catedrático de un instituto público, la falta de adaptación del profesorado secundario es un mal tan radical que sin resolverlo "será absolutamente inútil tantear cuantos planes de un bachillerato se nos ocurran [...], podrá algún día haber un plan de bachillerato, cuando haya una escuela de profesores de Instituto" (Ibidem, 333). En la misma línea de los institucionistas considera la imposibilidad de éxito en cualquier reforma que no cuente con un profesorado pedagógicamente preparado.

Toda esta argumentación a favor de la Pedagogía como componente indispensable en la preparación de los profesores de secundaria, no significa que don Rogerio deje esta disciplina libre de culpas. Al reflexionar sobre esta cuestión, se pregunta acerca del por qué del descrédito de la Pedagogía entre la mayoría de los profesores de la segunda enseñanza y concluye que:

Si se ha de ser justos, hay que convenir en que, si por curiosidad un profesor de Instituto echaba mano de tal o cual manual que de teorias pedagógicas trataba, se le servían lo más comúnmente unos recetarios semivacíos, rayanos casi siempre en el absurdo y a base de una tan deplorable cultura que solía arrojarse con desprecio aquel libro infame, confundiendo en el merecido desdén a lo que era un hondo y grave problema con lo que no significaba otra cosa que vanidad necia o mercaduría sórdida (Sánchez, 1922, n. ${ }^{\circ}$, 228).

Reconoce que, en su época, las cosas están cambiando y admite que no falta una discreta orientación nacional en estas cuestiones:

Pero de allá salió el descrédito de la pedagogía y el desdén irónico con que se han venido mirando estas hondas dificultades, por aquí casi siempre desfiguradas, merced a una preceptiva vacua, arbitraria y pedantesca. El profesor de instituto, licenciado o doctor, en posesión casi siempre de una superior cultura, que la de los dogmatizadores pedagógicos y falto de abundantes fuentes que le sugirieran verdaderos problemas, se burló, no sin razón, de ellos y de sus intentos, se afirmó en su cuantidad científica y tomó esta razón cuantitativa como suprema norma: el que más enseña, ese es el mejor profesor; y así se da el caso de ciertas cátedras de segunda enseñanza, descarnadas, frias, torturantes, en las cuales el maestro es un señor sabio a quien el alumno ve lejos, muy lejos de él, que sabe muchas cosas profundas y recónditas, asombro y susto del escolar de diez a quince años (lbidem).

\section{El temprano pensamiento de Rogerio Sánchez con relación a la formación del profesorado de enseñanza secundaria}

Es importante destacar que Rogerio Sánchez se habia mostrado partidario de la formación pedagógica con anterioridad a la fundación de La Segunda Enseñanza. En 1916, este catedrático de Lengua y Literatura en el instituto San Isidro, dio una conferencia en la Unión Iberoamericana, titulada "Lo que podría ser un bachillerato". Posteriormente, se publicó con el título de "Sentido pedagógico del Bachillerato" con prólogo del entonces ex ministro de Instrucción Pública Faustino Rodríguez-San Pedro.

Esta conferencia, que se publicó en dos entregas, como ya vimos, en La Segunda Enseñanza, se volvió a recoger en el último número del año 1922, pero ahora con el título de "Antecedentes para la historia de un ensayo en la segunda enseñanza". Con algunas de las expresiones que utiliza en el título de este artículo (antecedentes de un ensayo), don 
José Rogerio quiere dejar constancia de que su conferencia fue dicha y publicada antes (en enero de 1916) del RD de mayo de 1918, por el que se creó en Madrid el InstitutoEscuela. En consecuencia, también las ideas alli expresadas sobre la formación del profesorado de la segunda enseñanza son anteriores a dicha creación.

Por Real decreto de mayo de 1918 se creaba en Madrid, siendo Ministro el Excmo. Sr. D. Santiago Alba, el InstitutoEscuela. Quede bien puntualizado que en lo que hay de meritorio en esta creación del dicho Instituto-Escuela, el profesorado de Institutos fue su iniciador, puesto que un catedrático de Instituto indicó la conveniencia y aun urgencia de tal determinación, cuando a ella eran bien ajenas las iniciativas ministeriales y las de los que habian de dar forma a la nueva institución.

No había pensado el autor de este trabajo en reeditarlo; ejemplares del año 1916 hay aún muchos, pero requerido para que se coleccionen los documentos que pueden formar un día la historia de la actual Segunda enseñanza, ha seguido el consejo de varios compañeros.

Así, pues, lo que con el título Lo que podía ser un bachillerato se dijo y publicó hace seis años, sale ahora en nueva edición (Sánchez, 1922, n. 9, 455).

Se nota un cierto malestar en este profesor porque la experiencia educativa del Instituto-Escuela se haya llevado a cabo sin contar con los profesores de los institutos públicos. Cuando en el título utiliza la expresión "Antecedentes para la historia de un ensayo" queda clara su intención de precisar que su propuesta fue anterior al Real Decreto que creó el Instituto-Escuela.

Sin embargo, la originalidad reclamada tiene escaso fundamento, dado que, como ya se dijo en el apartado referido al entorno pedagógico, la preocupación de los institucionistas por la formación pedagógica de los docentes de la segunda enseñanza, puede situarse en el último cuarto del siglo XIX. El profesor A. Viñao data los "textos básicos que constituyen la expresión del ideario de la Institución Libre de Enseñanza en relación con la segunda enseñanza" en el período antes referido. El más antiguo sería Problemas de la segunda enseñanza publicado en 1892 (Viñao, 2000, 68), es decir, bastante antes de la citada conferencia (1916) de don José Rogerio. Cabe pensar que éste no hubiese leído a Giner, pero esta suposición parece difícil de sostener teniendo en cuenta la importancia y el alcance de la Ins- titución Libre de Enseñanza, en cuyo ideario se contenía el tema en cuestión.

\section{OTRAS FORMAS DE ESTIMULAR LA FORMACIÓN PEDAGÓGICA DEL PROFESORADO: INSTRUYENDO CON EL EJEMPLO}

Además de las opiniones sobre la formación pedagógica del profesorado expresadas en los artículos antes analizados, hay algunos textos que, sin aludir de forma expresa a dicha formación, sugieren, también, la necesidad de abordarla. En este sentido, puede hablarse de un discurso implícito, difuso, menos intencionado que el discurso directo que hemos venido analizando. Tal es el caso de varios artículos que se recogen en diversos números de la publicación, en los que se hace referencia a la manera en que distintas naciones europeas han afrontado esta cuestión.

En lo que respecta a este asunto hay que destacar un artículo publicado en el número 10 del año 1924 con el títuIo, "Formación pedagógica del Profesorado de Segunda enseñanza", en el que se describen los sistemas de formación pedagógica de Bélgica, Dinamarca, Holanda, Luxemburgo, Noruega y Polonia (Revista de Segunda Enseñanza, 1924, n. $\left.{ }^{10}, 34-40\right)$. El tema continúa en el número siguiente con la descripción de Checoslovaquia, Rumania y Francia (Revista de Segunda Enseñanza, 1924, n.11, 128-132). La lectura de estos breves informes nos permite concluir que en casi todos los países se cuenta con un seminario o una institución pedagógica donde se imparte formación profesional para los futuros docentes, de carácter teórico, durante un período que oscila entre uno y dos años. Esta tarea era supervisada por profesores en centros de enseñanza secundaria. Si bien no se hace comparación ${ }^{9}$, si se realiza una yuxtaposición que facilita el conocimiento. Éste podría actuar como elemento inspirador para que las autoridades nacionales de educación procedieran solucionando este ya viejo problema que era, por entonces, la formación pedagógica del profesorado de secundaria.

La mirada hacia Europa de los profesores vinculados a esta publicación, se pone de manifiesto, también, en las informaciones sobre los congresos internacionales. A algunos de ellos asistieron miembros del consejo de redacción de la revista. La temática de la formación pedagógica era, por

ARBOR Vol. 187749 mayo-junio [2011] 485-500 ISSN: 0210-1963 
entonces, una preocupación de carácter internacional que se vehiculaba muy especialmente en los congresos europeos; la publicación que analizamos daba cuenta de ello. Era otra manera de dar publicidad a la importancia de este tema, otra forma de divulgarlo. A lo largo de su existencia esta revista se hace eco de los congresos internacionales celebrados en Luxemburgo, Praga, Varsovia, Belgrado y Bucarest ${ }^{10}$. Entre estas reuniones, interesa destacar la de Praga, ya que en ella adquirió especial relevancia el tema que estudiamos. En las conclusiones de dicho congreso se destaca el hecho de que las discusiones se polarizaran en dos tendencias muy claras: la de los denominados pedagogistas, defendida por los belgas, y la de los universitarios, defendida por los franceses. Los primeros intentaban fundamentar en una base pedagógica la razón de una cultura científica; los universitarios, por su parte, expresaron su preocupación de un exceso de pedagogía que podría hacer desaparecer el espíritu de investigación y el cuidado en el cultivo científico. De ambos extremos surgió una tercera posición que se describe así:

El Congreso, después de haber oído las diferentes ideas expuestas acerca de la preparación pedagógica, estima que no es imposible conciliar aquellas opiniones. Juzga que la primera cualidad exigible a un profesor es la posesión de una sólida cultura general, unida al profundo conocimiento de su propia especialidad. Reconoce también que la preparación pedagógica hecha en la medida que se crea oportuna y según el espíritu, las tradiciones y necesidades de cada país, por una racional combinación del método filosófico, del método histórico y de aplicación práctica, puede favorecer y desarrollar la vocación pedagógica, cualidad indispensable al profesor... Se sobreentiende que una preparación pedagógica debe ser objeto de una remuneración especial (Revista de Segunda Enseñanza, n. ${ }^{\circ}$ 10, 1924, 17).

Dentro de este campo que hemos denominado "otras formas de estimular la formación pedagógica" parece interesante hacer alusión a un cierto número de artículos dedicados a mostrar, mediante descripción y, a veces, también con imágenes, algunos institutos nacionales y europeos. De esta manera, entendemos, podrian servir al público lector como patrón y como inspiración en aspectos tales como la arquitectura escolar, la organización de los centros, los materiales didácticos adecuados a las distintas asignaturas, las novedades en dichos aspectos, etc. Así nos encontramos, por ejemplo, con que bajo el título de
"Instituciones modelo", se describe el instituto de Aguilar y Eslava de la ciudad de Cabra, perteneciente a la provincia de Córdoba. La intencionalidad de servir de referente queda manifestada cuando el autor del artículo, el catedrático de Historia natural don Juan Carandell, dice que esa institución "demuestra de un modo patente, cómo debemos aspirar a desenvolver en España el grado de la enseñanza secundaria, si sinceramente queremos que nuestra obra, perdiendo algo del oropel de feria del cual solemos gustar los españoles, se transforme en oro de ley para el enriquecimiento de los valores morales de la Patria" (Revista de Segunda Enseñanza n. $\left.{ }^{\circ} 10,1924,21-34\right)$. Sigue una amplia y detallada descripción de la arquitectura, las instalaciones, las aulas y el material didáctico. Con la misma finalidad se describen otros como el de Álava (Ibidem, 1925, n. ${ }^{\circ} 16$, 441-446), el Instituto-Escuela (Ibidem, n. $17,511-516)$, el de Cáceres (Ibidem, 516-518).

En el número 11 de la revista se anuncia que en próximas publicaciones "se darán a conocer instituciones modelo que, dedicadas a la segunda enseñanza, florecen en el extranjero". Así nos encontramos con la del liceo Warocqué en Bélgica (Ibidem, 1925, n. 13, 211-213), entre otros. Parece interesante destacar la presentación de la Escuela Normal de Pisa en tanto fue una institución creada en 1813 , que tenía la finalidad de formar, justamente, al profesorado de la segunda enseñanza (Ibidem, 1927, n. ${ }^{\text {os }} 33$ y 34, 293-301).

\section{Orientaciones didácticas para la enseñanza de las ciencias}

Dada la sensibilidad pedagógica manifestada a lo largo de la revista por su fundador y por los profesores que participan en ella, no podían faltar los artículos dedicados a la didáctica y metodología de las ciencias. Las materias que tienen mayor protagonismo son la Historia natural, la Física y la Química, a cargo de los catedráticos Antonio Martínez (instituto San Isidro), José Estalella (instituto de Tarragona) y Jesús de la Puente (instituto de Barcelona). Destaca el profesor Estalella como colaborador asiduo.

La metodología planteada para la enseñanza de las materias científicas se inspira en los métodos experimentales, activos e intuitivos introducidos por los movimientos pedagógicos del último tercio del siglo XIX y comienzos del siglo XX: el movimiento de la Escuela Nueva y la in- 
novación pedagógica llevada a cabo en la Institución Libre de Enseñanza. Estos nuevos planteamientos pedagógicos renovadores influyeron en algunas de las reformas educativas que se produjeron a partir de 1918 en algunos paises europeos (Del Pozo, 2009, 209). De hecho, la revista se hace eco de esta renovación a través de la participación de su director en los congresos internacionales a los que asiste y publicita.

Se observan diferentes tendencias metodológicas para la enseñanza de las materias científicas. El profesor Antonio Martínez, catedrático de Historia natural del instituto San Isidro, señala una serie de estrategias didácticas con las que debe contar un profesor moderno para la enseñanza de la Historia natural: las clases prácticas en el laboratorio "donde el alumno hace ensayos mineralógicos, aguarda tallar una roca para disponerla a la observación microscópica, se familiariza con el manejo de la microscopia y confecciona alguna preparación micrográfica, tanto de Botánica como de Zoología y practica alguna disección" (Martínez, 1922, n. ${ }^{\circ}$ 2, 81). Otro tipo de recursos metode arte, etc. También se organizan excursiones científicas o interdisciplinares a los alrededores de la población. De esta manera, el alumno ve en la naturaleza lo que el profesor explicó en la cátedra y aprende a recolectar y a preparar lo recolectado, facilitando el conocimiento sin tener que recurrir sólo a la memoria (lbidem). Asimismo, se mencionan como medios auxiliares para las lecciones en la cátedra los aparatos de proyección fija y cinematográfica.

Algunos artículos en los que se describen excursiones y el aprendizaje que ellas motivaron, pueden considerarse, también, dentro de este ámbito que aquí hemos denominado "otras formas de estimular la formación pedagógica". Las excursiones se organizan como clases prácticas interdisciplinares sobre un centro de interés, que se analiza desde diversos aspectos culturales y científicos. Se plantea una enseñaza intuitiva, activa y vivencial, basada en lecciones de cosas extraídas del mundo real. Pueden ser diseñadas de forma interdisciplinar para que el aprendizaje sea más completo. Es una práctica que realizaban frecuentemente los institucionistas; también es recomendada por la redacción de la revista, invitando a los centros, profesores y alumnos -en fin, a los protagonistas- a que cuenten sus experiencias, crónicas, etc." ${ }^{11}$ dológicos son las visitas a museos científicos, históricos,
A modo de ejemplo, puede citarse la excursión realizada por el instituto San Isidro a la ciudad de Ávila, calificada de científica, histórica y artística, que se registra en el $n .{ }^{\circ} 6$ del año 1922 (La Segunda Enseñanza, n. ${ }^{\circ}$ 6, 1922, 322334). Alli se detalla el itinerario del viaje y todo lo que éste aportó como conocimiento para los alumnos. El programa empieza con las observaciones geológicas hechas, desde el tren, por el catedrático de Historia natural don Antonio Martínez. Éstas permitieron, por ejemplo, contemplar los sedimentos cuaternarios de la vertiente meridional de la sierra de Guadarrama y los campos de granito de dicha sierra. Ya en Ávila, el catedrático de Arte, el señor Vegue, invitado por los profesores, explica la historia de la ciudad visitando distintos sitios significativos como las murallas que rodean la ciudad, la casa de Santa Teresa, el monasterio de Santo Tomás, la catedral, etc. Sin duda esta descripción, con intención o no, muestra una estrategia didáctica que los lectores de la revista podían imitar y, seguramente, imitaron aquéllos preocupados por mejorar sus métodos.

Lo dicho antes es sólo un ejemplo de una forma de enseñanza y aprendizaje que los profesores del instituto de San Isidro ensayaron en repetidas ocasiones según puede constatarse en la revista que analizamos y que, como ya hemos señalado, consideramos una forma difusa de indicar caminos didácticos para solventar una formación pedagógica pobre 0 inexistente de sus colegas de la enseñanza pública. En un número de 1925 se da cuenta de una excursión realizada a Burgos. El programa del viaje es similar al seguido en Ávila y se realizan observaciones geográficas, del arte que encierra la ciudad. Éstas habían estado precedidas de "una reseña artística e histórica de la ciudad cabeza de Castilla", realizada por el "ilustre artista y profesor del San Isidro D. Marceliano Santamaría" (Santamaría, 1925, n. ${ }^{\circ} 16,465-480$ ).

Este tipo de enseñanza también es propuesta por otro catedrático que escribió en la revista, se trata de Juan Carandell (profesor del instituto Aguilar y Eslava de Cabra, Córdoba). Para la enseñanza de las Ciencias o Historia natural resulta imprescindible que el alumno conozca de forma activa e intuitiva la naturaleza. Cuando nos habla del Gabinete de historia natural del mencionado instituto, señala que muchas de sus colecciones entomológicas o de insectos, aves, minerales, herbarios, etc. proceden de los trabajos de los alumnos. Este profesor utiliza las ex-

ARBOR Vol. 187749 mayo-junio [2011] 485-500 ISSN: 0210-1963

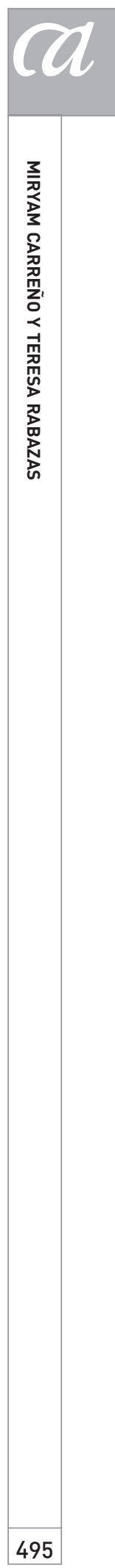

doi: 10.3989/arbor.2011.749n3003 
cursiones como medio para observar la naturaleza, ya que argumenta que el entorno natural enseña mucho más que las lecciones de un manual. En la revista se recogen algunas fotografías de las colecciones que tenía el instituto en 1924 (Carandell, 1926, n. ${ }^{\circ} 19,15-20$ ).

Las enseñanzas científicas requerían de la práctica de los laboratorios para verificar y comprobar algunas de las teorias expuestas en los libros de texto o en las lecciones explicadas en clase. Se aconsejaba una enseñanza práctica e intuitiva que favoreciera el aprendizaje por observación, experimentación y comprobación personal del alumno: "Lo que el escolar descubre, inventa y construye es elemento esencial de su conciencia" (Feliú Egidio, 1922, n. ${ }^{\circ}$ 8, 398).

En relación con la enseñanza experimental de las ciencias, en los artículos de la revista observamos dos tendencias. Por un lado, la reclamación de una mayor dotación presupuestaria para la adquisición de material didáctico y científico y, por otro, la propuesta de soluciones prácticas ante los limitados medios con los que contaba la enseñanza pública, como es la simplificación del material didáctico y la autoconstrucción de dicho material, por parte de los profesores y alumnos, con materiales de desecho o de fácil acceso. Para Estalella los escasos recursos con que contaban los institutos, no debían constituir un impedimento para el aprendizaje por descubrimiento, tan necesario en la enseñanza de las ciencias.

En relación con la simplificación del material didáctico, el profesor Estalella propone ejemplos de dicho material para el aprendizaje de ciertos conceptos de la Física. Entiende que el profesorado de secundaria debería tener en cuenta el principio de máxima simplificación: "Cabría establecer este principio aplicable a la enseñanza de toda ciencia: dar al instrumental la mayor simplificación posible; cuando basten los dedos no usen los instrumentos; cuando se puede trabajar con objetos corrientes no acuda a aparatos especiales". Enumera múltiples ejemplos de principios de la física con aparatos e instrumentos utilizados en la vida ordinaria (veletas, huertos, aparatos eléctricos, etc.). Intenta demostrar en sus artículos que la falta de experimentación nunca puede excusarse por la pobreza de medios. El origen de los conocimientos científicos está en la investigación, basada en la observación y en la experimentación y no en la explicación de una lectura. Ni el libro, ni el profesor pueden nunca sustituir la actividad del estudiante.
Todo conocimiento debe basarse en la investigación. No se trata de demostrar leyes en el laboratorio (aprendizaje deductivo), sino de aprenderlas y quizás mejor descubrirlas, contribuyendo al aprendizaje por descubrimiento. En este caso, el papel del profesor es muy análogo al del guía que acompaña al excursionista. El estudiante ha de sentir la creación del conocimiento. La conexión entre la vida real y la escuela es algo que reclaman todos los profesores de enseñanzas científicas (Estalella, 1925, 563-588; Ibidem, 1926, n. $\left.{ }^{\circ} 20,63-81\right)$.

El paso siguiente a la simplificación sería la autoconstrucción del material por parte de los alumnos con ayuda del profesor, utilizando materiales de desecho o aprovechando objetos del entorno. Se demuestra con ejemplos (veánse figs. 2 y 3), cómo construir una balanza de laboratorio, un soporte universal barato, una palanca, un embudo de separación, una centrifugadora manual, diferentes objetos y material construidos por los alumnos a partir de materiales de desecho, inservibles o rotos (bombillas, alambre, tornillos, vasos, hierro, etc.) (De la Puente, 1925, n. ${ }^{\circ} 17$, 502-503; 1926, n. ${ }^{\circ} 19,13-14 ; 1926$, n. $\left.21,107-109\right)$. En opinión del catedrático José Estalella, no pueden faltar en un laboratorio los aparatos históricos que él piensa deben ser imprescindibles: tubo de Mariotte y el soporte de corrientes móviles de Ampère. Si no se tienen, el profesor verá la manera de suplirlos (Estalella, 1926, n. ${ }^{\circ} 22$, 164-166).

La adquisición de material didáctico por parte de los institutos es otra de las preocupaciones que se observa en la revista. Así, por ejemplo, el San Isidro da cuenta, en un artículo, de la compra de un aparato de proyección fija de la casa Leitz, utilizado para la proyección microscópica horizontal (véase fig. 1), y la compra de un modelo cinematográfico por incandescencia de la casa Pathé. La utilidad didáctica de este aparato consiste en que se puedan mostrar determinados objetos (preparaciones micrográficas, postales instructivas, escritos, objetos pequeños) a toda una clase, que permita al profesor al mismo tiempo que se proyecta el objeto, hacer la explicación pertinente. Se pretende solventar uno de los problemas que tienen algunos institutos, como es la masificación de alumnos en sus aulas. También resulta muy útil para preparar las excursiones ya que permite al profesor mostrar diapositivas, grabados, planos, mapas, etc. sobre los lugares que se van a visitar, y así señalar los aspectos más relevantes, explicar el itinerario, etc. El profesor Antonio Martínez Ilega a 


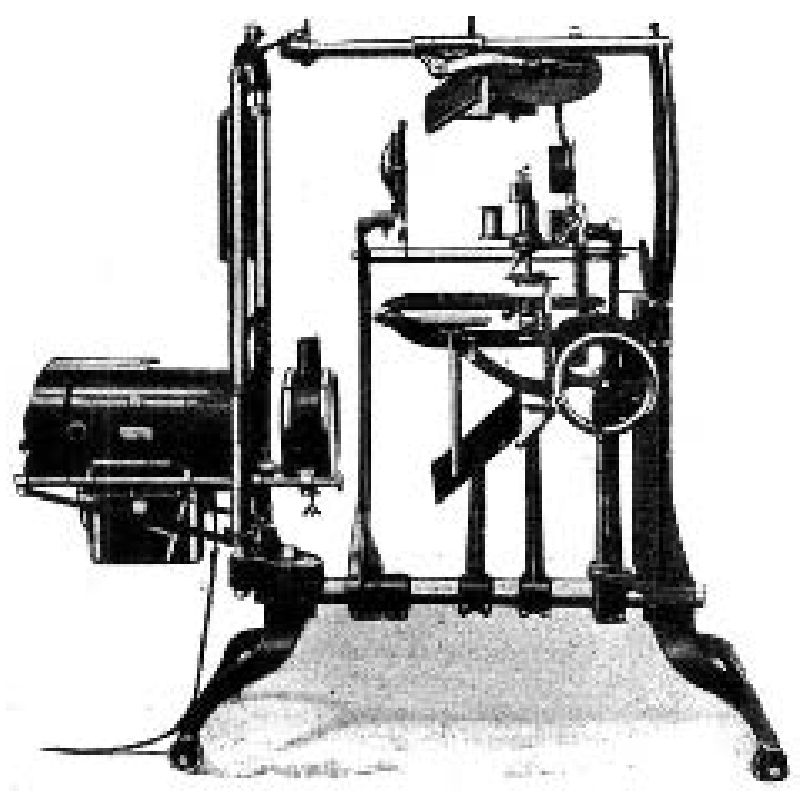

Figura 1. Aparato de proyección microscópica horizontal fïa de la casa Leitz. En Martínez, Antonio (1924). Los aparatos de proyección $y$ los centros de enseñanza. Revista de Segunda Enseñanza, Año II, n. ${ }^{\circ} 10,124-126$.

sugerir, en este artículo, que los institutos podrian ponerse de acuerdo para intercambiar películas instructivas y economizar recursos (Martínez, 1924, n. 10, 124-126).

\section{Conclusiones}

La revista aquí analizada surge en los inicios del siglo XX en un contexto de inquietudes reformistas con respecto al nivel de la segunda enseñanza. Este tema ocupó buena parte de sus páginas. Más allá de las reformas legislativas referidas a la estructura de este nivel -que también fue un objetivo de la publicación-, las mayores preocupaciones manifestadas en sus artículos hicieron referencia a la necesidad de innovar la formación del profesorado, incluyendo en ella conocimientos de carácter pedagógico.

Este tema atrajo a los profesores de la segunda enseñanza más comprometidos con la educación; ellos fueron los protagonistas de una severa crítica con respecto a la
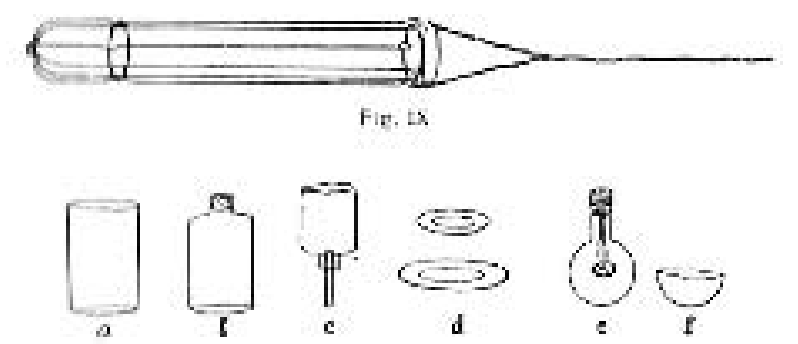

$\operatorname{rig} \mathbf{X}$

Figura 2. Ejemplos de autoconstrucción del material. En De la Puente, Jesús (1925). El material de nuestras clases. Revista de Segunda Enseñanza, Año $I V, n .^{\circ} 19,14$.
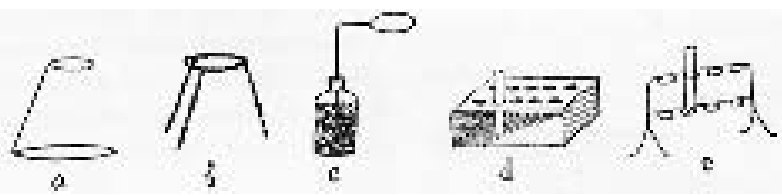

Figura 3. Ejemplos de autoconstrucción del material. En De la Puente, Jesús (1926). El material de nuestras clases. Revista de Segunda Enseñanza, Año $V, n .^{\circ} 21,107$.

que consideraban defectuosa formación pedagógica de los profesores de este nivel.

Los educadores que se reunieron en torno a la revista aquí analizada fueron, dentro de la enseñanza pública, los más entusiastas abanderados de esta innovación y los más activos en su justificación y defensa. Sin duda constituyeron una élite que destacó notablemente en el grupo mayoritario de indiferentes y hasta contrarios a dicha formación. El cierre de la revista, según hemos señalado en páginas anteriores, por falta de apoyo económico e institucional, nos da pautas acerca tanto de la indiferencia mayoritaria como de los esfuerzos y el empeño de sus promotores.

A pesar de las dificultades antes anotadas, La Segunda Enseñanza tuvo una buena difusión tanto dentro como fuera de España, como puede comprobarse a través del análisis de sus números. Fue, pues, una publicación de carácter internacional apreciada y elogiada por los profesores del entorno europeo según hemos demostrado en este estudio.

ARBOR Vol. 187749 mayo-junio [2011] 485-500 ISSN: 0210-1963 
Esta publicación constituyó un medio de transmisión de la necesidad de esa formación pedagógica tan discutida entonces y cuestionada, incluso, hasta el dia de hoy. Fue también, por eso mismo, un testimonio de la conciencia que algunos profesores de la enseñanza pública tenían de la importancia de estos conocimientos para los licenciados que pretendian orientar su actividad en el nivel de la segunda enseñanza.

Constituyó, además, una buena plataforma de divulgación de ideas y experiencias educativas innovadoras con respecto a la enseñanza secundaria. Se convirtió en un excelente vehículo de propuestas de renovación pedagógica en la práctica profesional del profesorado de los institutos. Canalizó propuestas y reflexiones de algunos profesores sobre los problemas que los afectaban.

No cabe duda acerca del esfuerzo del pequeño sector de profesores que se agrupó en torno a la revista analizada, por incorporar a la formación del profesorado de la enseñanza secundaria pública las tendencias innovadoras de principios de siglo, que se caracterizaban por considerar los conocimientos pedagógicos como una parte imprescindible en la preparación de los futuros docentes. Pero esta iniciativa chocó con la indiferencia de buena parte de los destinatarios de esa formación, con la escasa acogida que las corrientes renovadoras tuvieron entre las autoridades educativas, con dificultades económicas.

Más allá del poco éxito que tuvo esta iniciativa de innovación pedagógica, el conocimiento de su peripecia histórica nos ayuda a la reflexión sobre un tema que, como entonces, ha vuelto a ser materia de debates y hasta de enfrentamientos entre partidarios y detractores de esta formación no ajenos, por cierto, a intereses corporativos. Como los institucionistas, el grupo de profesores que sostuvieron esta publicación no creían en reforma alguna que no estuviera precedida por una seria formación de unos profesores identificados con su cometido.

\section{NOTAS}

1 Contraportada del primer número de la revista. Alli se aclara, también, que las suscripciones deben hacerse en las librerias de Perlado, Páez y Cía., en la calle Arenal, 11 y en la de Victoriano Suárez en la calle Preciados, 48. Se pone en conocimiento de los lectores que no se editará en julio y agosto.

2 El motivo de la suspensión fue dejar paso a una nueva revista destinada a ser portavoz de la Asociación de Catedráticos de Instituto que no llegó a concretarse.

3 Alfonso Navarro Jurado expresa una opinión similar en su tesis doctoral sobre la Historia del instituto de segunda enseñanza San Isidro de Madrid (1845-1936). Considera que la vida efímera de la publicación se debió a razones de naturaleza económica ya que se pretendió financiar a través de los suscriptores y de las instituciones de secundaria pero la precariedad económica de éstas fue una dificultad insalvable. Esta tesis -inédita- fue defendida en 1991 en la Universidad Complutense (p. 803).

4 Se refiere a la reforma de E. Callejo de 25 de agosto de 1926.

5 La Segunda Enseñanza, Año I, n. 2 , 1922, p. 65.

6 Sobre este plan veáse: Díaz de la Guardia, E., Evolución y desarrollo de la enseñanza media en España de 1875 a 1930. Un conflicto político pedagógico, Madrid, CIDE, 1988, especialmente los capítulos 6 y 7 . El plan de estudios había sido simplificado por RD de 6 de octubre de 1903 con el ministro Gabino Bugallal.

7 El profesor A. Viñao Frago señala los textos básicos en los que se manifiesta el ideario de la ILE en relación a la segunda enseñanza en su trabajo: "Un modelo de reforma educativa: los
Recibido: 1 de marzo de 2010

Aceptado: 30 de junio de 2010 
institutos-escuela (1918-1936)". En Boletín de la Institución Libre de Enseñanza, II Época, 39, 2000, pp. 63-88.

8 Giner de los Ríos, F., Nota sobre la segunda enseñanza. Citado en, Seage, J.; Guerrero Salom, E. y Quintana de Uña, D., Una pedagogía de la libertad. La Institución Libre de Enseñanza, Madrid, Cuadernos para el Diálogo, 1977, p. 236.

9 La Educación comparada no era entonces una disciplina desarrollada. Daba sus primeros pasos en un ámbito geográficamente limitado al mundo anglosajón.

10 La revista informa de los congresos internacionales vinculados a la segunda enseñanza en lo que respecta a lugar y fecha de celebración, requisitos de inscripción, temática, conferenciantes, etc. Estos eventos se solian celebrar en el mes de agosto con la finalidad de permitir la participación de la mayoría del profesorado europeo. En 1922 se celebró el congreso de Luxemburgo y al año siguiente, 1923, el de Praga, en 1924 el de Varsovia, en 1925 el de Belgrado, en 1926 el de Ginebra y en 1927 el de Bucarest. El director de la revista que analizamos fue participante activo en muchos de estas reuniones de las cuales informó a través de ella.

11 En este sentido, señalamos algunas crónicas de los lugares visitados por varios profesores y alumnos de secundaria: en Francia se visitaron algunos liceos franceses, en donde se describen los edificios y los métodos de enseñanza, basados en una enseñanza viva y cíclica, sin utilizar libros de texto, practicando, en cambio, la lectura comentada de clásicos; de esta manera, se favorecía el diálogo entre profesores y alumnos. También se relatan excursiones a Andalucía, en las que se visitan Escuelas
Normales, monumentos, etc. Destacamos las excursiones organizadas por el instituto San Isidro a modo de ejemplo: Ávila, Toledo, Alcalá de Henares, El Escorial, puertos de Guadarrama, de Navacerrada y de Cotos, etc. Otra experiencia similar es relatada por un profesor del instituto Aguilar y Eslava de Córdoba, en la que da cuenta de algunas visitas a los alrededores, como Ronda, Cuenca del Guadiana, Algeciras y Gibraltar. Por último, señalamos la realización de visitas a fábricas en las que el alumno pudo conocer de cerca el proceso de fabricación de algunos productos químicos, el vidrio, el aluminio, la pólvora, los textiles y otros tejidos. Se visitaron, también, talleres de alfarería, jabonería, herrería, tintorería, hornos de cal, de cemento, ladrillería, etc.

\section{BIBLIOGRAFÍA}

Adellac, Miguel (1922): "Formación del profesorado de institutos", La Segunda Enseñanza, Año I, n. ${ }^{\circ}$ 1, pp. 5-8.

Actas del Congreso Pedagógico HispanoPortugués-Americano (1892): Trabajos preparatorios del Congreso. Resúmenes generales, Madrid, Librería de la Viuda de Hernando.

Carandell, Juan (1925): "Excursión escolar a Córdoba, Sevilla, Huelva y Ríotinto. Instituto Aguilar y Eslava", Revista de Segunda Enseñanza, Año III, n. 16, pp. 452-462.

Carandell, Juan (1926): "El Museo de Historia Natural del Instituto Aguilar y Eslava", Revista de Segunda Enseñanza, Año IV, n. 19, pp. 15-20.

De la Puente, Jesús (1925): "El material de nuestras clases", Revista de Segunda Enseñanza, Año III, n. 17, pp. 502503.
De la Puente, Jesús (1926): "El material de nuestras clases", Revista de Segunda Enseñanza, Año IV, n. ${ }^{\circ} 19$, pp. 13-14.

De la Puente, Jesús (1926): "El material de nuestras clases", Revista de Segunda Enseñanza, Año IV, n.21, pp. 107109.

De Puelles Benitez, M. (1999): Educación e ideología en la España contemporánea, Madrid, Tecnos.

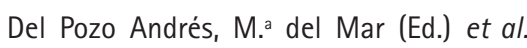
(2009): Teorías e instituciones contemporáneas de educación, Madrid, Biblioteca Nueva (2. ${ }^{\text {a }}$ ed.).

Díaz de la Guardia, E. (1988): Evolución y desarrollo de la enseñanza media en España de 1875 a 1930. Un conflicto político pedagógico, Madrid, CIDE.

Estalella, José (1925): "La simplificación del material escolar de Física y Química", Revista de Segunda Enseñanza, Año III, n. ${ }^{\circ} 18$, pp. 563-588.

Estalella, José (1926): "Simplificaciones en la experimentación química", Revista de Segunda Enseñanza, Año IV, n. ${ }^{\circ} 20$, pp. 63-81.

Estalella, José (1926): "Un gabinete escolar", Revista de Segunda Enseñanza, Año IV, n. ${ }^{\circ} 22$, pp. 164-166.

"Excursión a Burgos hecha por Profesores y alumnos del Instituto de San Isidro, durante los días 10, 11, 12, 13 y 14 de abril de 1925", La Segunda Enseñanza, n. ${ }^{\circ} 16,1925$, pp. 465-480.

Feliú Egidio, Vicente (1922): "El laboratorio en la segunda enseñanza", La Segunda Enseñanza, Año I, n. ${ }^{\circ}$ 8, pp. 398-405.

La Segunda Enseñanza, Año I, n. ${ }^{\circ}$ 6, 1922, pp. 322-334.

Martínez, Antonio (1922): "La enseñanza de la Historia Natural en los Institutos Generales y Técnicos", La Segunda Enseñanza, Año I, n. ${ }^{\circ}$ 2, pp. 79-85.

Martínez, Antonio (1924): "Los aparatos de proyección y los centros de enseñanza", Revista de Segunda Enseñanza, Año II, n. ${ }^{\circ} 10$, pp. 124-126. 
"Nuestra presentación", en El Instituto, Año I, n. ${ }^{\circ} 1,1928$.

"Nuestra revista y su significación", La Segunda Enseñanza, Año I, n.o 5, 1922, pp. 242-243.

Puelles Benítez, M. de. (1999): Educación e ideología en la España contemporánea (1767-1975), Madrid, Tecnos.

Revista de Segunda Enseñanza, n. ${ }^{\circ} 11$, Año II, 1924, pp. 128-132.
Revista de Segunda Enseñanza, Año I, n. ${ }^{\circ} 25,1926$.

Revista de Segunda Enseñanza, n. ${ }^{\circ}$ 10, Año II, 1924.

Sánchez Rogerio, José (1922): "Sentido pedagógico del bachillerato", La Segunda Enseñanza, Año I, n. ${ }^{\circ} 4$, pp. 225-229.

Sánchez Rogerio, José (1922): "Sentido pedagógico del bachillerato", La Segunda Enseñanza, Año I, n. ${ }^{\circ}$, pp. 335-340.
Silió, C. (1922): "Pedagogía Nacional", La Segunda Enseñanza, Año I, n. ${ }^{\circ}$ 1, p. 4. Tuñón de Lara, M. (1981): La España del siglo XX, tomo 1, Madrid, Alianza.

"Una explicación", El Instituto, Año I, n. ${ }^{\circ s} 3$ y 4,1928, pp. 129-130.

Viñao Frago, A. (2000): "Un modelo de reforma educativa: los institutos-escuela (1918-1936)", Boletín de la Institución Libre de Enseñanza, II Época, 39, pp. 63-88. 